\title{
A Differential Oligopoly Game with Differentiated Goods and Sticky Prices $^{1}$
}

\author{
Roberto Cellini* - Luca Lambertini** \\ * Dipartimento di Economia e Metodi Quantitativi \\ Università di Catania \\ Corso Italia 55, 95129 Catania, Italy \\ fax 39-095-370574 \\ e-mail cellini@mbox.unict.it \\ ** Dipartimento di Scienze Economiche \\ Università degli Studi di Bologna \\ Strada Maggiore 45, 40125 Bologna, Italy \\ fax 39-051-2092664 \\ e-mail lamberti@spbo.unibo.it
}

May 29, 2002

\footnotetext{
${ }^{1}$ We thank Caterina Colombo for helpful comments and discussion. The usual disclaimer applies.
} 


\begin{abstract}
We investigate a dynamic oligopoly game where goods are differentiated and prices are sticky. We study the open-loop and the closed-loop Nash equilibrium, and show that the latter equilibrium entails a larger level of steady state production as compared to the former; both equilibria entail a larger level of production in steady state than the static game. We also study the effects of price stickiness and product differentiation upon the steady state equilibrium allocation. We find that per-firm equilibrium output is increasing in both product differentiation and price stickiness.
\end{abstract}

JEL Classification: D43, D92, L13

Keywords: differential games, loop decision rules, product differentiation, price stickiness. 


\section{Introduction}

The aim of this note consists in studying the properties of the equilibria in a dynamic oligopoly model with price stickiness, along the lines first introduced by Simaan and Takayama (1978) and then extended by Fershtman and Kamien (1987), and Cellini and Lambertini (2000). The novelty of this paper rests on the fact that we analyse an oligopoly where goods are differentiated, and we study how good differentiation and price stickiness interact in shaping the equilibrium allocation.

We take into consideration both the open-loop and the closed-loop Nash equilibrium. In both cases, an economically meaningful symmetric steady state exists; this equilibrium is a saddle. We focus on the steady state equilibrium allocation, and study its determinants. The already known properties of the differential game involving homogenous goods are confirmed; in particular, (i) the static game entails a lower level of production as compared to the steady-state equilibrium production levels of the differential game, and the steady-state Nash equilibrium production under the open-loop information structure is smaller than under the closed-loop rule; (ii) the stickier are the prices, the higher the steady state Nash equilibrium production levels. This consistency is not surprising, provided that the homogenous oligopoly case can be interpreted as a particular case of the present model.

In addition, we show that the degree of differentiation among goods is effective in determining both the production levels, and the responsiveness of quantities to price stickiness. In particular, we show that the higher is the (symmetric) degree of differentiation among goods, the lower is the steady state level of production. Moreover, the higher is the degree of differentiation, the lower is the sensitivity of the steady state level of production to the price stickiness. This means that the degree of differentiation and the degree of price stickiness affect the steady state equilibrium level of production in much the same way.

The outline of the paper is as follows. Section 2 introduces the basics of the model. Section 3 develops the differential game under the open-loop information structure, while Section 4 solves the closed-loop game. Both Sections focus on the steady-state level of production, showing that the steady state is a saddle, and presenting comparative statics exercises on the production levels. Section 5 concludes the paper. 


\section{The setup}

A simple way to model price stickiness is to imagine that price adjusts, in response to the difference between its "notional" level and its current level. Under this perspective, price can be seen as the state variable of a dynamic system. Only a part of the difference between the "notional" and the "current" level of price can be corrected, in the presence of stickiness. This can be motivated, for instance, by costly adjustment. We formalise this idea, borrowing from Simaan and Takayama (1978), the following motion low concerning the price of any good $i:^{1}$

$$
\frac{d p_{i}(t)}{d t} \equiv \dot{p}_{i}(t)=s_{i}\left\{\widehat{p}_{i}(t)-p_{i}(t)\right\}
$$

where $\widehat{p}_{i}(t)$ denotes the notional level of price of good $i$ at time $t$, while $p_{i}(t)$ denotes its current level. Notice that the speed of adjustment is captured by parameter $s_{i}$, with $0<s_{i}<1$. The lower is $s_{i}$, the higher is the degree of price stickiness.

As far as the notional price concerns, it is dictated by the demand condition, deriving from the preference structure of consumers. We assume that the notional price in any instant $t$ is defined as follows:

$$
\widehat{p}_{i}(t)=A(t)-B q_{i}(t)-D \sum_{j \neq i} q_{j}(t)
$$

This function is borrowed from Spence (1976) and employed by Singh and Vives (1984), Vives (1985), Lambertini (1997), Cellini and Lambertini (1998, $2002)$, inter alia. The number of available varieties is assumed to be constant over time and equal to $N$, with $i \in[1, N]$. Parameter $A$ measures the market size or the reservation price, which is assumed to be equal across varieties for the sake of simplicity. As for parameters $B$ and $D$, assume $0 \leq D \leq B$. Notice that parameter $D$ captures the degree of substitutability between any pair of different goods. In the limit case $D=0$, goods are independent and each firm becomes a monopolist. In the opposite limit case $D=B$, the goods produced by different firms are perfect substitutes and the model collapses

\footnotetext{
${ }^{1}$ See also Fershtman and Kamien (1987). Mehlmann (1988, ch. 5) provides an exhaustive exposition of both contributions.Fershtman and Kamien (1990), and Tsutsui and Mino (1990) present further results on the same model, in the case of a finite horizon.
} 
into the homogeneous oligopoly model. Thus, the higher is parameter $D$, the lower is the (symmetric) degree of differentiation. ${ }^{2}$

Consider a population of $N$ single-product firms. The instantaneous production cost function of firm $i$ is assumed to be quadratic:

$$
C_{i}(t)=c_{i} q_{i}(t)-\frac{1}{2}\left[q_{i}(t)\right]^{2}, 0<c_{i}<A .
$$

As a consequence, the instantaneous profit function of firm $i$ is:

$$
\pi_{i}(t)=q_{i}(t) \cdot\left[p_{i}(t)-c_{i}-\frac{1}{2} q_{i}(t)\right] .
$$

For future reference, we report the solution of the static problem, where the notional price is equal to the current price, and any firm chooses the quantity to be produced. In such a case, the maximisation of the function: $\pi_{i}=q_{i} \cdot\left[A-B q_{i}-D \sum_{j \neq i} q_{j}\right]-c_{i} q_{i}-\frac{1}{2} q_{i}^{2}$, with respect to $q_{i}$, and then the imposition of the symmetry conditions $q_{i}=q_{j}=q$ lead to find the following symmetric Cournot-Nash equilibrium quantity:

$$
q_{C N}=\frac{(A-c)}{[1+2 B+D(N-1)]}
$$

In the problem we are interested here, however, the current price of any good is generally different from its notional level. The production decisions of firms affect notional prices, but current prices evolve subject to the existence of price stickiness. We assume that firms choose the quantity to be produced, so that we are in a Cournot framework. More precisely, each player (i.e., each firm) chooses the path of his control variable $q_{i}(t)$ over time, from the present to infinity, i.e., $t \in[0, \infty)$, in order to maximize the present value of the profit flow, subject to (i) the motion laws regarding the state variables, and (ii) the initial conditions. Formally, the problem of player $i$ may be written as follows:

$$
\begin{aligned}
\max _{q_{i}(t)} J_{i} & =\int_{0}^{\infty} e^{-\rho_{i} t} q_{i}(t) \cdot\left[p_{i}(t)-c_{i}-\frac{1}{2} q_{i}(t)\right] d t \\
\text { s.t. } & : \frac{d p_{i}(t)}{d t}=s_{i}\left\{\widehat{p}_{i}(t)-p_{i}(t)\right\}, i \in[1, N] \\
\text { s.t. } & : \quad p_{i}(0)=p_{i, 0}, \quad i \in[1, N]
\end{aligned}
$$

\footnotetext{
${ }^{2} \mathrm{~A}$ model where $D$ is a variable, whose dynamics is driven by the investment efforts of firms devoted to product differentiation is in Cellini and Lambertini (2002).
} 
Notice that the number of the state variables in the problem of each player $i$ is equal to $N$, corresponding to the price of the $N$ available varieties, while the control variable is one for each player, specifically, the quantity to be produced. The factor $e^{-\rho_{i} t}$ discounts future gains, and the discount rate $\rho_{i}$ is assumed to be constant.

We solve the problem by considering -in turn- the open-loop solution, and the closed-loop memoryless solution.

\section{The open-loop solution}

Here we look for the open-loop Nash equilibrium, i.e., we examine a situation where firms commit to a production plan at $t=0$ and stick to that plan forever.

The Hamiltonian function is:

$$
\begin{aligned}
\mathcal{H}_{i}(t)= & e^{-\rho_{i} t} \cdot\left\{q_{i}(t) \cdot\left[p_{i}(t)-c_{i}-\frac{1}{2} q_{i}(t)\right]+\right. \\
& +\lambda_{i}^{i}(t) s_{i}\left[A-B q_{i}(t)-D \sum_{j \neq i} q_{j}(t)-p_{i}(t)\right]+ \\
& \left.+\sum_{j \neq i} \lambda_{j}^{i}(t) s_{j}\left[A-B q_{j}(t)-D \sum_{h \neq j} q_{h}(t)-p_{j}(t)\right]\right\}
\end{aligned}
$$

where $\lambda_{i}^{i}(t)=\mu_{i}^{i}(t) e^{\rho_{i} t}$, and $\mu_{i}^{i}(t)$ is the co-state variable associated by player $i$ to the price of his product, $p_{i}(t)$; similarly, $\lambda_{j}^{i}(t)=\mu_{j}^{i}(t) e^{\rho_{i} t}$, with $\mu_{j}^{i}(t)$ being the co-state variable associated by player $i$ to the price of the good $j \neq i$. As usual, supplementary variables $\lambda$ represent co-states in current value, and are introduce to ease calculation.

The outcome of the open-loop game is summarised by the following:

Proposition 1 When the open-loop Nash equilibrium solution concept is adopted, a symmetric steady state exists, where the individual output and the market price are:

$$
\begin{aligned}
q_{O L}^{\infty} & =\frac{(s+\rho)(A-c)}{(s+\rho)[1+B+D(N-1)]+s B} \\
p_{O L}^{\infty} & =A-\frac{\lfloor B+D(N-1)\rfloor(s+\rho)(A-c)}{(s+\rho)[1+B+D(N-1)]+s B}
\end{aligned}
$$


Such a steady state is a saddle.

Proof. In order to find the open-loop Nash equilibria, we have to solve the following first-order condition:

$$
\frac{\partial \mathcal{H}_{i}(t)}{\partial q_{i}(t)}=0
$$

along with the adjoint equations

$$
\begin{array}{r}
-\frac{\partial \mathcal{H}_{i}(.)}{\partial p_{i}(t)}=\frac{\partial \lambda_{i}^{i}(t)}{\partial t}-\rho \lambda_{i}^{i} \\
-\frac{\partial \mathcal{H}_{i}(.)}{\partial p_{j}(t)}=\frac{\partial \lambda_{j}^{i}(t)}{\partial t}-\rho \lambda_{j}^{i}
\end{array}
$$

The first order condition and the adjoint equations have to be considered along with the initial conditions $\left\{p_{i}(0)=p_{i, 0}\right\}_{i=1}^{N}$ and the transversality conditions, which set the final value of the state and/or co-state variables:

$$
\lim _{t \rightarrow \infty} \lambda_{i}^{i}(t) \cdot p_{i}(t)=0, \lim _{t \rightarrow \infty} \lambda_{j}^{i}(t) \cdot p_{j}(t)=0 .
$$

$>$ From (10),(11) and (12) we obtain respectively:

$$
\begin{gathered}
q_{i}(t)=p_{i}(t)-c_{i}-\lambda_{i}^{i}(t) s_{i} B-D \sum_{j \neq i} \lambda_{j}^{i}(t) s_{j} \\
\frac{\partial \lambda_{i}^{i}(t)}{\partial t}=\left(s_{i}+\rho_{i}\right) \lambda_{i}^{i}(t)-q_{i}(t) \\
\frac{\partial \lambda_{j}^{i}(t)}{\partial t}=\left(s_{j}+\rho_{i}\right) \lambda_{j}^{i}(t)
\end{gathered}
$$

Now we introduce some symmetry assumptions, so that we focus on symmetric equilibria. In particular we assume: $s_{i}=s_{j}=s ; \rho_{i}=\rho ; c_{i}=c$. Moreover, in the symmetric equilibrium, $q_{i}=q_{j}=q$, and $p_{i}=p_{j}=p$. Eventually, we pose $\lambda_{i}^{i}=\lambda_{\text {own }}$, and $\lambda_{j}^{i}=\lambda_{\text {other }}$ for any $j \neq i$. This amounts to stating that each player attaches a co-state variable to the price of his own product, and a co-state variable to the price of any other variety. It is worth stressing that we can not postulate $\lambda_{\text {own }}=\lambda_{\text {other }}$, since the effect of the price of the variety produced by each firm on his own profit, is obviously different from the effect of the price of the varieties produced by the opponents. 
Differentiating equation (14) w.r.t. time, and taking into account the symmetry assumptions (so that $i$ and $j$ subscripts and superscripts can be omitted), we obtain:

$$
\frac{d q(t)}{d t}=\frac{d p(t)}{d t}-s B \frac{d \lambda_{\text {own }}(t)}{d t}-(N-1) s D \frac{d \lambda_{\text {other }}(t)}{d t}
$$

The dynamic constraint and equations (15) and (16) rewrite, respectively, as follows:

$$
\begin{gathered}
\frac{d p(t)}{d t}=s[A-B q(t)-D(N-1) q(t)-p(t)] \\
\frac{d \lambda_{\text {own }}(t)}{d t}=(s+\rho) \lambda_{\text {own }}(t)-q(t) \\
\frac{d \lambda_{\text {other }}(t)}{d t}=(s+\rho) \lambda_{\text {other }}(t)
\end{gathered}
$$

This means that, for any player, the dynamics of all relevant variables may be described by a system of four dynamic equations. The system can be written in matrix form as follows:

$$
\left[\begin{array}{c}
\dot{p}(t) \\
\dot{q}(t) \\
\dot{\lambda}_{\text {own }}(t) \\
\dot{\lambda}_{\text {other }}(t)
\end{array}\right]=\left[\begin{array}{cccc}
-s & -s[B+D(N-1)] & 0 & 0 \\
1 & 0 & -s B & -(N-1) s D \\
0 & -1 & (s+\rho) & 0 \\
0 & 0 & 0 & (s+\rho)
\end{array}\right]\left[\begin{array}{c}
p(t) \\
q(t) \\
\lambda_{\text {own }}(t) \\
\lambda_{\text {other }}(t)
\end{array}\right]
$$

It easy to show that a (non-trivial) steady state does exist in this dynamic system. We denote by $p^{\infty}, q^{\infty}, \lambda_{\text {own }}^{\infty}, \lambda_{\text {other }}^{\infty}$ the steady state levels of the relevant variables, namely, the price, the output level, and the co-state variables associated with the own price and with the price of different varieties, respectively. From equation (17) it is immediate to note that if $d p / d t=0$, $d \lambda_{\text {own }} / d t=0, d \lambda_{\text {other }} / d t=0$ hold simultaneously, then $d q / d t=0$. From equation (19) we note that $d \lambda_{\text {own }} / d t=0$ entails $\lambda_{\text {own }}^{\infty}=q^{\infty} /(s+\rho)$, while from equation (20) we note that $d \lambda_{\text {other }} / d t=0$ entails $\lambda_{\text {other }}^{\infty}=0$. Equation (18) shows that $d p / d t=0$ entails $p^{\infty}=A-[B+D(N-1)] q^{\infty}$. Using equation (14) under the symmetry conditions, we obtain:

$$
q_{O L}^{\infty}=\frac{(s+\rho)(A-c)}{(s+\rho)[1+B+D(N-1)]+s B}
$$


Simple substitutions permit us to fully characterize the steady state market allocation under the open loop information structure:

$$
\begin{aligned}
p_{O L}^{\infty} & =A-\frac{[B+D(N-1)](s+\rho)(A-c)}{(s+\rho)[1+B+D(N-1)]+s B} \\
\lambda_{\text {own }}^{\infty} & =\frac{(A-c)}{(s+\rho)[1+B+D(N-1)]+s B}
\end{aligned}
$$

As a final point, we have to investigate the stability property of the steady state. We already know that, in the case of homogenous oligopoly, the steady state is a saddle, completely described by a dynamic system of 2 differential equations in 2 variables, namely price and quantity (see Cellini and Lambertini, 2000). In the present, more general, setting with a differentiated oligopoly, four variables (and four differential equations) are necessary to fully characterise the dynamics of the system - even under the particular case of the symmetric equilibrium. In order to have that the steady state is stable in the saddle point sense, it is sufficient that exactly two out of the four characteristic roots of the Jacobian matrix associated with the dynamic system (that is, the Jacobian in system (21)), have a negative real part. This is the case indeed in the problem at hand: two out of four characteristic roots of the Jacobian matrix in (21) are real and positive; the other two roots have negative real parts. ${ }^{3}$ This condition implies that the steady state is stable in the saddle point sense. This means that given the initial level of price $p(0)=p_{0}$ and the condition $\lambda_{\text {other }}(0)=0$ (which also satisfies the transversality condition), one can find initial values $q(0)>0$ and $\lambda_{\text {own }}(0)>0$, such that the system converge to the steady state $\left(p^{\infty}, q^{\infty}, \lambda_{\text {own }}^{\infty}, \lambda_{\text {other }}^{\infty}\right)$. This concludes the proof.

Notice that the present result encompasses the result from the model with product homogeneity. ${ }^{4}$ Notice also that the steady state level of production, under the open-loop information structure is larger than its counterpart in the static Cournot game. This is easily proved, by confronting (22) with (5). Consistently, the steady state level of price is smaller in the dynamic game as

\footnotetext{
${ }^{3}$ One characteristic root is $(s+\rho)$; the expressions of remaining three roots are rather heavy. One of them is real and positive; the two remining roots are complex, with negative real parts.

${ }^{4}$ In fact, when $D=B=1$ the level of $q_{O L}^{\infty}$ given by equation (22) of the present paper coincides with the steady state level of production in the homogenous oligopoly model presented by Cellini and Lambertini (2000) - see in particular their equation (12).
} 
compared to the static game. This result is well known in the literature (see, e.g., Simaan and Takayama, 1978, Reynolds, 1987, Piga, 2000, inter alia). It means that the prolonged time of non-cooperative interaction leads to higher levels of production (and lower prices) as compared to static interaction.

Simple comparative statics exercises on the steady state level of production $q_{O L}^{\infty}$ lead to the following results, holding for all $N>1$.

(i) $\partial q_{O L}^{\infty} / \partial D<0$ : the higher is $D$, that is, the higher is the substitutability among goods (and the lower is the differentiation) the smaller is the steady state level of production in the symmetric open-loop Nash equilibrium. The intuition behind this result could be provided by the following argument: a higher substitutability among varieties means a smaller demand for any individual firm, and hence lower market power. Consequently, the reaction of firms leads to an equilibrium where production is lower.

(ii) $\partial q_{O L}^{\infty} / \partial s<0$ : the lower is the price stickiness (i.e., the larger is $s$ ), the smaller is the steady state production; put differently, the stickier are the prices, the larger the production in steady state is. This result is well known in the literature on differential oligopoly games with sticky prices (see also Piga, 2000; Cellini and Lambertini, 2000). A rough intuition for this result is provided by the following argument: when prices are sticky, the current production levels of firms are weakly effective in moving current prices; this fact leads firms to high levels of current and future production. On the contrary, when prices move largely in response to production decisions, firms choose to shrink the output levels.

(iii) $\partial^{2} q_{O L}^{\infty} /(\partial s \partial D)>0$ : this cross derivative measures how the sensitivity of steady state production to differentiation is affected by price stickiness. In this respect, note that the higher is $s$, i.e., the less sticky are prices, the higher is the sensitivity of production with respect to differentiation. Since differentiation and price stickiness have the same effect on production, their mutual interaction strengthens the effect on individual equilibrium output.

\section{The closed-loop solution}

Under the closed-loop information structure, firms do not precommit on any path and their strategies at any instant may depend on all the preceding history. In this situation, the information set used by firms in setting their strategies at any given time is often simplified to be only the current value of 
the state variables at that time. The relevant equilibrium concept is in this case is labelled closed-loop memoryless Nash equilibrium; it is strongly time consistent (see, e.g., Dockner et al., 2000).

The outcome of the closed-loop game is summarised by the following:

Proposition 2 When the closed-loop memoryless Nash equilibrium solution concept is adopted, a symmetric steady state exists, where the individual output and the market price are:

$$
\begin{aligned}
q_{C L}^{\infty} & =\frac{\Psi(s+\rho)(A-c)}{\Psi[(s+\rho)(1+B+D(N-1))+s B]-(N-1) s^{2} D^{2}} \\
p_{C L}^{\infty} & =A-[B+D(N-1)] q_{C L}^{\infty}
\end{aligned}
$$

where $\Psi \equiv \rho+s(1+B+D(N-2))$. Such a steady state is a saddle.

Proof. The relevant Hamiltonian function is still (9), while the first order condition and the adjoint equations for the player $i$ are as follows:

$$
\begin{gathered}
\frac{\partial \mathcal{H}_{i}(t)}{\partial q_{i}(t)}=0 \\
-\frac{\partial \mathcal{H}_{i}(.)}{\partial p_{i}(t)}-\sum_{h \neq i} \frac{\mathcal{H}_{i}(.)}{\partial q_{h}(t)} \frac{\partial q_{h}^{*}(t)}{\partial p_{i}(t)}=\frac{\partial \lambda_{i}^{i}(t)}{\partial t}-\rho_{i} \lambda_{i}^{i} \\
-\frac{\partial \mathcal{H}_{i}(.)}{\partial p_{j}(t)}-\sum_{h \neq i} \frac{\mathcal{H}_{i}(.)}{\partial q_{h}(t)} \frac{\partial q_{h}^{*}(t)}{\partial p_{j}(t)}=\frac{\partial \lambda_{j}^{i}(t)}{\partial t}-\rho_{i} \lambda_{j}^{i}
\end{gathered}
$$

Also in this case the first order condition and the adjoint equations have to be considered along with the initial conditions $\left\{p_{i}(0)=p_{i, 0}\right\}_{i=1}^{N}$ and the transversality conditions (13).

The terms

$$
\frac{\partial \mathcal{H}_{i}(.)}{\partial q_{h}(t)} \frac{\partial q_{h}^{*}(t)}{\partial p_{j}(t)}
$$

appearing in (26) and (27) capture strategic interaction, in any instant of time, through the feedback from states to controls, which is by definition absent under the open-loop solution concept. Whenever the expression in (28) is zero for all $j$, then the closed-loop memoryless equilibrium collapses into the open-loop Nash equilibrium (see, e.g., Driskill and McCafferty, 1989); this is not the case in the present setting. 
In order to solve this problem, we take into account that $\frac{\partial q_{h}^{*}(t)}{\partial x_{j}(t)}=1 \mathrm{iff}$ $h=i$ while $\frac{\partial q_{h}^{*}(t)}{\partial x_{j}(t)}=0$ otherwise, and $\frac{\partial q_{h}^{*}(t)}{\partial p_{j}(t)}=1$ iff $h=j$ while $\frac{\partial q_{h}^{*}(t)}{\partial p_{j}(t)}=0$ otherwise. From $(25),(26)$ and (27) we obtain respectively:

$$
\begin{gathered}
q_{i}(t)=p_{i}(t)-c_{i}-\lambda_{i}^{i}(t) s_{i} B-D \sum_{j \neq i} \lambda_{j}^{i}(t) s_{j} \\
\frac{\partial \lambda_{i}^{i}(t)}{\partial t}=\left(s_{i}+\rho_{i}\right) \lambda_{i}^{i}(t)-q_{i}(t) \\
\frac{\partial \lambda_{j}^{i}(t)}{\partial t}=\left(\rho_{i}+s_{i}\right) \lambda_{j}^{i}(t)+s_{i} D \lambda_{i}^{i}(t)+D \sum_{h \neq i, j} \lambda_{h}^{i}(t) s_{i}+s_{h} B \lambda_{h}^{i}(t)
\end{gathered}
$$

Now we introduce the usual symmetry assumptions, in order to focus on the symmetric equilibria. In particular, $s_{i}=s_{j}=s_{h}=s ; \rho_{i}=\rho ; c_{i}=c$; furthermore, $q_{i}=q_{j}=q$, and $p_{i}=p_{j}=p$. Moreover, we pose $\lambda_{i}^{i}=\lambda_{\text {own }}$, and we postulate the symmetry assumption $\lambda_{j}^{i}=\lambda_{h}^{i}=\lambda_{\text {other }}$ for any $j \neq i, h \neq i$. These assumptions permit us to write equation (29) as follows:

$$
q(t)=p(t)-c-s B \lambda_{o w n}(t)-s D(N-1) \lambda_{\text {other }}
$$

In the symmetric steady state the following relationships hold:

(i) from (26): $\lambda_{\text {own }}^{\infty}=q^{\infty} /(\rho+s)$;

(ii) from (27): $\lambda_{\text {other }}^{\infty}=-s D \lambda_{\text {own }}^{\infty} /[\rho+s(1+B+D(N-2))]$;

(iii) from the dynamic constraint: $p^{\infty}=A-[B+D(N-1)] q^{\infty}$.

Subsequent substitutions into equation (32) lead to find the following relation holding in steady state:

$$
q^{\infty}=A-B q^{\infty}-D(N-1) q^{\infty}-c-\frac{s B q^{\infty}}{(\rho+s)}+\frac{s^{2} D^{2}(N-1) q^{\infty}}{[\rho+s(1+B+D(N-2)](\rho+s)}
$$

so that the steady state level of production, under the symmetric closed-loop memoryless Nash equilibrium turns out to be:

$$
\begin{aligned}
q_{C L}^{\infty} & =\frac{\Psi(s+\rho)(A-c)}{\Psi[(s+\rho)(1+B+D(N-1))+s B]-(N-1) s^{2} D^{2}} \\
\Psi & \equiv \rho+s(1+B+D(N-2))
\end{aligned}
$$


In the remainder of the Section we discuss, in turn, the dynamic properties of the steady state, and the role of the parameters in determining the steady state production under the closed-loop information structure, as compared to the open-loop and the static game Nash equilibria.

As far the first point is concerned, the dynamic system (under symmetry) can be written in matrix form as follows:

$$
\begin{gathered}
{\left[\begin{array}{c}
\dot{p}(t) \\
\dot{q}(t) \\
\dot{\lambda_{\text {own }}(t)} \\
\lambda_{\text {other }}(t)
\end{array}\right]=\theta\left[\begin{array}{c}
p(t) \\
q(t) \\
\lambda_{\text {own }}(t) \\
\lambda_{\text {other }}(t)
\end{array}\right],} \\
\theta=\left[\begin{array}{cccc}
-s & -s[B+D(N-1)] & 0 & 0 \\
1 & 0 & -s B & -(N-1) s D \\
0 & -1 & (s+\rho) & 0 \\
0 & 0 & 0 & \lfloor s+\rho+s B+D(N-2)]
\end{array}\right]
\end{gathered}
$$

Also in this case, it is possible to find the characteristic roots of the Jacobian matrix in (35), and it is possible to check that exactly two out of the four characteristic routs have negative real parts. Therefore, the steady state is stable in the saddle point sense - that is, given the initial conditions, the steady state is reached only for one appropriate combination of the control variable and co-state variables, at the initial time. This dynamic feature of the steady state is consistent with the analogous result in the particular case of homogeneous oligopoly, where the $2 \times 2$ dynamic system presents a steady state, which is a proper saddle (see Cellini and Lambertini, 2000, Section 5). This concludes the proof.

Simple comparative statics exercises on the steady state level of production $q_{C L}^{\infty}$ lead to the following points, confirming all the substantial conclusions about the steady state production under the open-loop information structure: for all $N>1$, (i) $\partial q_{C L}^{\infty} / \partial D<0$; (ii) $\partial q_{C L}^{\infty} / \partial s<0$; (iii) $\partial^{2} q_{O L}^{\infty} /(\partial s \partial D)>0$.

In this oligopoly with differentiated goods, like in the homogenous good case, the steady state level of production turns out to be larger under the closed-loop information structure, than under the open-loop, as the comparison between equations (22) and (34) makes clear. Both levels are larger than the production of the static Cournot game. This fact can be explained on the 
following grounds. The closed-loop output level is higher than the open-loop output level because, taking into account feedback effects, each firm tries to preempt the rivals. Since this holds for all firms alike, the outcome is that the closed-loop steady state production exceeds the open-loop steady state production. ${ }^{5}$ In turn, the open-loop steady state output exceeds the static (or myopic) output because in the static game there is no time for adjustment and therefore firms have no way of trying to overproduce in order to preempt the rivals.

As a consequence, from the firms' viewpoint, the static situation (or, a situation where firms are myopic) is the most profitable one. On the contrary, the steady state allocation in the closed-loop memoryless equilibrium is socially preferred both to the open-loop steady state and to the static equilibria.

Fershtman and Kamien (1987, pp. 1159-61) also investigate the properties of the limit game, where the speed of adjustment $s$ tends to infinity. They show that, in such a case, the open-loop equilibrium coincides with the Nash equilibrium of the static game. However, considering an infinitely high speed of price adjustment seems more a mathematical curiosum than a theoretically relevant case, in that whenever $s>1$, the instantaneous change in price is larger than the error $\widehat{p}(t)-p(t)$. If we consider the case $s=1$ as the limit case, we can check that, in such a situation, both $q_{O L}^{\infty}$ and $q_{C L}^{\infty}$ are larger than the static Cournot-Nash output for all $N>1$ and all $\rho>0$.

Finally, as the number of firms becomes infinitely large, optimal individual output tends to zero independently of the solution concept. As the market becomes perfectly competitive, open-loop and closed-loop steady state solutions coincide with the static Cournot-Nash solution, which is itself reproducing the perfectly competitive outcome.

\section{Concluding remarks}

In this paper we have investigated the properties of a dynamic oligopoly game with sticky prices and differentiated products. It is important to stress that the rigidities we have dealt here with, are real rather than nominal, provided that we have taken a partial equilibrium approach, with sticky relative prices.

\footnotetext{
${ }^{5}$ This is usually observed when firms control variables are output levels, investment levels, etc. (see, e.g., Reynolds, 1987).
} 
We have shown that the dynamic rule governing the price motion (and in particular the degree of price stickiness) affect the final allocation, i.e., the steady state under the Nash equilibrium of the dynamic game. In particular two properties are worth mentioning: (i) in the (subgame perfect) closed-loop memoryless Nash equilibrium a steady state exist, which is stable in the saddle point sense, where the production is larger and the price is lower as compared to the open-loop steady state solution; (ii) irrespective of the equilibrium concept one adopts, in the dynamic framework, the steady state output levels and price levels are, respectively, higher and lower then their counterparts in the static game. Property (i) can be reformulated by saying that, if firms are unable to initially commit to a given output plan for the whole time horizon, then subgame perfection entails overproduction (for analogous results see Spence, 1979; and Reynolds, 1987). Property (ii) suggests that the dynamic nature of interaction leads forms to over-produce, as compared to the Nash equilibrium of a static interaction.

The above mentioned results are analogous with the findings from the homogeneous oligopoly model. In the present paper, additional results have been found, concerning the effects of the differentiation among the goods produced by firms upon steady state allocations. Under both the open-loop and the closed-loop solution concepts, the higher is the substitutability among goods, the lower is the steady state level of production; the tougher is the price stickiness, the higher the steady state level of production. Under this respect, the degree of price stickiness and the degree of product differentiation exert the same qualitative effects on the steady state output level. 


\section{References}

[1] Basar, T. and G.J. Olsder (1982, 1995²), Dynamic Noncooperative Game Theory, San Diego, Academic Press.

[2] Cellini, R., L. Lambertini (2002) "A Differential Game Approach to Investment in Product Differentiation", Journal of Economic Dynamics and Control, forthcoming.

[3] Cellini, R., L. Lambertini and G. I. P. Ottaviano (1998), "Differentiated Oligopoly and Product Innovation: An Endogenous Growth Approach", mimeo, University of Bologna.

[4] Dockner, E.J, S. Jørgensen, N. Van Long, G. Sorgen (2000), Differential Games in Economics and Managment Science, Cambridge University Press, Cambridge.

[5] Dockner, E.J., G. Feichtinger and S. Jørgensen (1985), "Tractable Classes of Nonzero-Sum Open-Loop Nash Differential Games: Theory and Examples", Journal of Optimization Theory and Applications, 45, 179-97.

[6] Driskill, R. and S. McCafferty (1989), "Dynamic Duopoly with Adjustment Costs: A Differential Game Approach", Journal of Economic Theory, 69, 324-38.

[7] Fershtman, C. (1987), "Identification of Classes of Differential Games for Which the Open-Loop is a degenertaed Feedback Nash Equilibrium", Journal of Optimization Theory and Applications, 55, 217-31.

[8] Fershtman, C. and M.I. Kamien (1987), "Dynamic Duopolistic Competition with Sticky Prices", Econometrica, 55, 1151-64.

[9] Fershtman, C. and M.I. Kamien (1990), "Turnpike Properties in a Finite-Horizon Differential Game: Dynamic Duopoly with Sticky Prices", International Economic Review, 31, 49-60.

[10] Fershtman, C., M. Kamien and E. Muller (1992), "Integral Games: Theory and Applications", in Feichtinger, G. (ed.), Dynamic Economic Models and Optimal Control, Amsterdam, North-Holland, 297-311. 
[11] Mehlmann, A. (1988), Applied Differential Games, New York, Plenum Press.

[12] Piga, C. (2000), "Competition in a Duopoly with Sticky Price and Advertising", International Journal of Industrial Organization, 18, 595-614.

[13] Reinganum, J. (1982), "A Class of Differential Games for Which the Closed Loop and Open Loop Nash Equilibria Coincide", Journal of Optimization Theory and Applications, 36, 253-62.

[14] Reynolds, S.S. (1987), "Capacity Investment, Preemption and Commitment in an Infinite Horizon Model", International Economic Review, $\mathbf{2 8}, 69-88$.

[15] Simaan, M. and T. Takayama (1978), "Game Theory Applied to Dynamic Duopoly Problems with Production Constraints", Automatica, 14, 161-6.

[16] Spence, A.M. (1976), "Product Differentiation and Welfare", American Economic Review, 66, 407-414.

[17] Spence, A. M. (1979), "Investment Strategy and Growth in a New Market", Bell Journal of Economics, 10, 1-19.

[18] Starr, A.W. and Y.C. Ho (1969), "Nonzero-Sum Differential Games", Journal of Optimization Theory and Applications, 3, 184-208.

[19] Tsutsui, S. and K. Mino (1990), "Nonlinear Strategies in Dynamic Duopolistic Competition with Sticky Prices", Journal of Economic Theory, 52, 136-61. 